\title{
The essentials of diabetes prevention and management during COVID-19 pandemic
}

\section{ABSTRACT}

Diabetes cases are soaring at alarming rates globally, majorly type 2 diabetes, with $90 \%$ of cases while the Africa and Asia continents will be the epicenter and battlegrounds for the condition in the coming years. Presently, the world is faced with a coronavirus disease (COVID-19) pandemic, and similar to many other diseases, diabetes shares negative associations with COVID-19 in terms of disease severity and outcomes, some of which are longer hospitalization, higher risk of mortality, longer periods of recovery, higher odds of admission into the intensive care unit (ICU), and higher risk of severe illnesses in association with other co-morbidities.

To this end, there is a need for an efficient diabetes prevention and management plan that is generally accepted among all populations worldwide during the pandemic and afterward. In this respect, this narrative review provides a broad overview on research detailing effective preventive and management alternatives against diabetes, and findings affirmed that engaging in regular physical activities, adequate intake of dietary fiber, intake of total plant foods, quitting and avoiding smoking, maintaining a healthy weight, avoiding the intake of alcohol, avoiding fats and sugary laden diets, avoiding drugs and medications that cause diabetes, controlling blood pressure and cardiovascular diseases, infection prevention, regular medical checkup and screening, and prevention of depression and stress will

Address for correspondce:

Israel Oluwasegun Ayenigbara

Department of Health Education,

University of Ibadan,

Post Office Street Number 022, Postal Code 200284,

Ibadan, Nigeria,

e-mail: histrealite2647@gmail.com

Clinical Diabetology 2021, 10; 6: 429-437

10.5603/DK.2021.0052

Received: 9.04.2021

Accepted: 14.09.2021 offer beneficial effects in the prevention of diabetes, preventing the progression of diabetes among the high-risk population, and the prevention of complications among diabetes patients during and after the pandemic. (Clin Diabetol 2021; 10; 6: 429-437)

Key words: diabetes, prevention, management, SARS-COV2, COVID-19, pandemic period

\section{Introduction}

Diabetes, which is usually a long-term medical condition occurs when the pancreas cannot make a sufficient quantity of insulin that is required, or when the body fails to efficiently make use of the insulin produced [1]. Diabetes affects approximately 463 million people globally as of 2019 , with $90 \%$ of the confirmed cases being type 2 [2]. In this regard, the main focus of this review is on ways to prevent, or slow the occurrence of diabetes, as well as the effective management of diabetes globally during the coronavirus disease (COVID-19) pandemic periods and beyond.

\section{Sources of literature selections}

To accomplish the main objectives of this review, the PubMed, Goggle scholar, Medline, and relevant national and global health databases (the World Health Organization (WHO), Center for Disease Control and Prevention (CDC), International Diabetes Federation (IDF), and American Diabetes Association (ADA) electronic websites were searched comprehensively between the periods of May 2020 - February 2021 to obtain scientific data and viable literature on the various ways that can be applied to prevent and manage diabetes.

Information on the global occurrence of diabetes

Over 425 million people were living with diabetes globally in 2017, an increase from around 382 million 
and 108 million people in 2013 and 1980 respectively, while diabetes affects approximately $8.8 \%$ of the older populations globally, an increase from $4.7 \%$ in 1980 [3-5].

The commonest type of diabetes is type 2 diabetes, which accounts for $90 \%$ of the confirmed cases worldwide [6, 7]. Although some statistics show the rates of diabetes to be evenly divided across gender, more cases of type 2 diabetes are recorded from the male population [7-9].

Also, excessive blood glucose which is a major indication of diabetes results in around 2.2 million deaths of people before the age of 70 years globally $[1,5,10]$. In 2017, the IDF calculated that diabetes, directly and indirectly, resulted in 4 million deaths globally, while diabetes was the seventh leading cause of death globally in 2016 as per the WHO [1, 3].

Diabetes, which is a global health condition, especially type 2 is more predominant specifically in developed nations. However, worryingly, there has been an exponential increase in type 2 diabetes cases in underdeveloped and developing nations over the previous years, where over $80 \%$ of diabetes mortalities are recorded, while in the coming years, there will be an exponential increase of diabetes cases on the continents of Africa and Asia [5, 11, 12].

One estimate indicates that diabetes increases the chances of early death, almost twofold, while in 2019, over 4.2 million deaths were caused by diabetes, and the global financial cost for the treatment and management of diabetes patients in 2017 was approximately US\$727 billion [1, 2].

In addition, findings from a specific country's estimations revealed that the United States (US) expended over 327 billion US dollars on diabetes alone in 2017, while the average medical costs for diabetes patients are three times higher when compared to other conditions $[13,14]$.

The findings from this section underscore the need for viable measures and continued sensitization programs by primary health care practitioners, various governmental health agencies, and specific nongovernmental organizations in different countries to prevent the future occurrences of diabetes, and more importantly during the pandemic period because the global rates of diabetes cases are expected to increase by $48 \%$ in the periods of 2017 to 2045 [3].

\section{The association between diabetes} and COVID-19

The novel type of the coronavirus disease called COVID-19, which was first detected in China in the latter part of 2019, has spread to almost all countries and regions of the world. Because of the sporadic mode of transmission, and severe nature of the disease, COVID-19 was declared a major world public health problem, and subsequently a pandemic by the WHO [15].

As in the case of many other diseases, COVID-19 shares numerous negative associations with diabetes in terms of disease severity and outcomes. For example, diabetes patients infected with COVID-19 face a $7.3 \%$ chance of mortality from any other COVID-19-associated illness [16].

In addition, findings from a meta-analysis study of COVID-19 patients with diabetes on hospital admission in China indicated a death rate of $9.9 \%$, while diabetes was additionally associated with worse recovery outcomes [17]. Furthermore, findings from a pooled analysis on the association of diabetes and COVID-19 severity and mortality revealed that prior diabetes patients face twice the risk of severe outcomes and mortality from COVID-19 than patients without diabetes [18]. To this end, it is imperative that COVID-19 patients who are also diabetic should be frequently watched by treating physicians [18].

Likewise, in the current pandemic, diabetics face higher chances of admission into the intensive care unit (ICU) for COVID-19 [19]. It is necessary to note that the care of diabetes in the ICU is often demanding and complex, however, the situation will be more complicated if a diabetes patient is admitted into the ICU for COVID-19 [19].

Oftentimes, diabetes predisposes people to other chronic conditions, consequently making the impact of COVID-19 possibly even more severe. For instance, findings from a systematic review and meta-analysis on the effects of numerous co-morbidities on severe outcomes from COVID-19 revealed that severe outcomes were evident in $526(13.16 \%)$ of COVID-19 patients, and the presence of high blood pressure, diabetes, heart disease, and serious kidney condition had a positive correlation in COVID-19 patients experiencing severe outcomes from the disease, while diabetes alone had a significant effect on mortality in people with COVID-19 [20].

In addition, research findings from a similar study on the relationship of obesity and diabetes to COVID-19 events in Mexico revealed that early diabetes, obesity, chronic obstructive pulmonary disease, increasing age, high blood pressure, immune suppression, and serious kidney problems increases significantly the risk of severe events and deaths in COVID-19 patients, while obesity mediates the impact of diabetes on the severity of COVID-19 49.5\% of the time [21].

Furthermore, early diabetes increases the odds of COVID-19 hospital admissions, while obesity increases the chances of admission into the ICU for COVID-19 and the need for a ventilator or assistive breathing [21]. In addition to the above findings, the predictive 


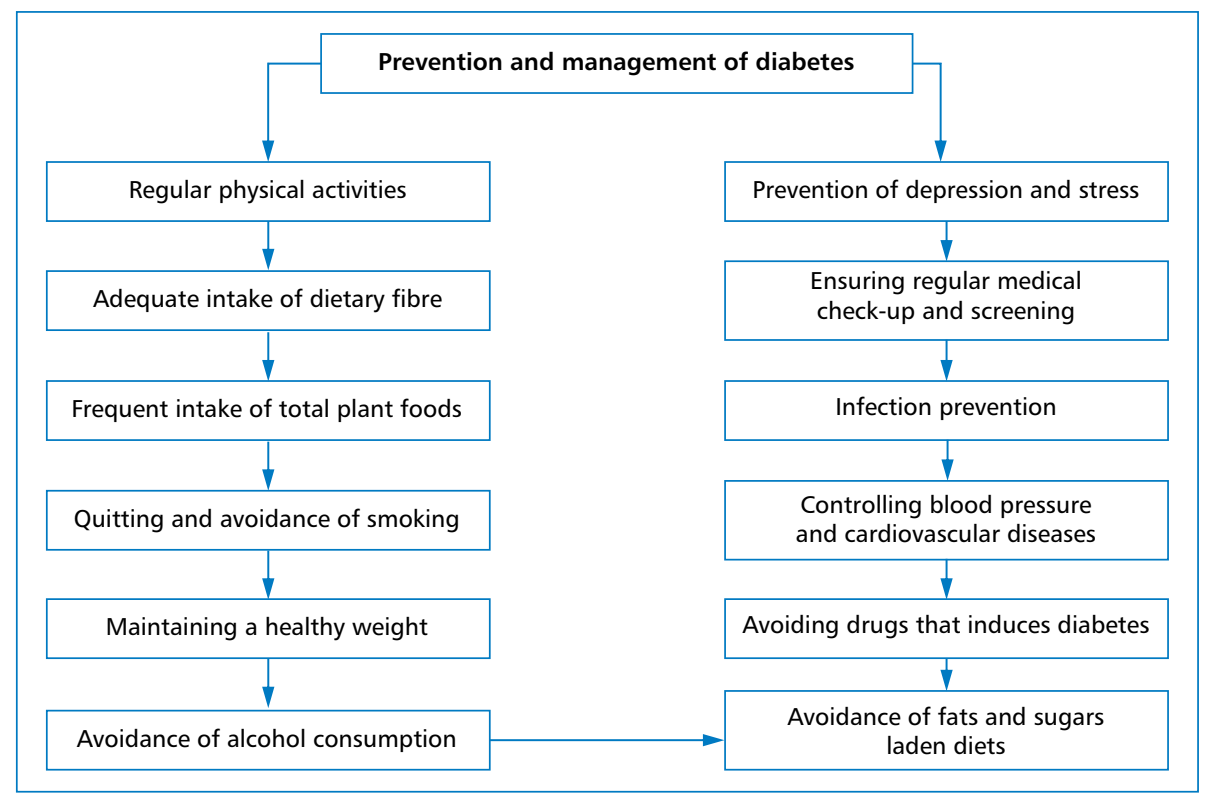

Figure 1. A layout for the prevention and management of diabetes

factors for the severity of COVID-19 as observed by the researchers were advancing age $(\geq 65)$ and diminishing age $(<40)$, early and late diabetes cases, obesity, serious kidney problems, high blood pressure, and immune suppression [21].

The early insights from this section firmly indicate the many severe outcomes associated with diabetes if infected with COVID-19. This is possible because when diabetes patients are infected by a viral pathogen, it is usually complex to treat them due to the presence of a possible unstable serum glucose status, coupled with the manifestations of other diabetes complications due to impairments of the immunity, thereby making it difficult for the body to wade off the pathogen, and possibly making recuperation more challenging and lengthier due to inflammation [22].

In addition, the viral pathogen also develops and thrives well in cells with increased or concentrated levels of serum glucose [22]. In this respect, if serum glucose is balanced and managed effectively, and in addition to the absence of diabetes-associated health conditions for example heart problems, the impact and severity of COVID-19 on diabetes patients will be reduced and may even be on par with people without the condition [22].

\section{Evidence-based prevention and management guidelines for diabetes}

Over the years, some lifestyle-centered diabetes preventive measures have proven minimal effectiveness in mitigating and slowing the incidence of the condition as evident in the rising numbers of cases globally.
Even more important is the severity of morbidity and mortality that comes along with the infection of COVID-19. To this end, this section discusses state-ofthe-art findings and applicable novel ways available in literature till now, on the prevention and management of diabetes (Fig. 1).

\section{Engaging in frequent physical activities}

There is strong proof of an inverse relationship between physical activities participation and a reduced incidence of type 2 diabetes due to the effects of physical activities in the reduction of fatty tissues, while all categories of high and low impact physical activities are helpful in this regard [23].

In addition, physical activity helps in insulin production, lowers the concentration of glucose in the blood, and diminishes the risk of cardiovascular diseases [24]. For significant health outcomes, not less than 150 minutes weekly of low or high-impact physical activities are required [24, 25].

Another finding from a meta-analysis on physical activity and the occurrence of gestational diabetes indicates that physical activities indices, for example (walking) prior-to pregnancy, and in the periods of pregnancy, and (participating in high impact physical activities) prior-to pregnancy were helpful in preventing gestational diabetes [26].

Notably, routine engagement in physical activities offers many beneficial effects to pregnant women [27]. These includes but is not limited to a reduction in the incidence of glucose metabolic disorder, which helps 
to maintain a healthy weight, reduces pain in the lower back region, and prevents the occurrence of type 2 diabetes after delivery [27].

Furthermore, physical activities also help against prolonged labor, reduce complications during child delivery, reduce depression, tiredness, anxiety, and stress which correlates with positive wellbeing during pregnancy [27]. Physical activities additionally reduce the incidence of hypertension during pregnancy (preeclampsia) and prevent stillbirths and premature births [27].

To this end, in order to reduce and maintain a healthy weight during the pandemic, thereby preventing diabetes and other associated non-communicable chronic diseases, indoor physical exercises are advised and essential when outdoor physical activities are not permitted or allowed because of lockdowns and restrictions.

\section{Adequate intake of dietary fiber}

Findings from available large data sets suggest that the consumption of a substantial quantity of dietary fiber, usually (> $25 \mathrm{~g} / \mathrm{d}$ for women and $>38 \mathrm{~g} / \mathrm{d}$ for men) reduces the occurrence of type 2 diabetes by 20-30\% [28].

In addition, a higher intake of carbohydrates fiber in combination with a reduced saturated and trans fat diet offers beneficial effects in the prevention of type 2 diabetes among high-risk people by drastically reducing and preventing weight and fat gain $[29,30]$.

Furthermore, the consumption of dietary fiber reduces the incidence of chronic diseases by helping with the balancing of blood glucose levels, thereby lowering the odds of heart disease as evident in populations with a higher intake of dietary fibers [31].

Specifically, the incidence of type 2 diabetes, specific cancers, and cardiovascular diseases are diminished, while healthy body weight could also be achieved through higher consumption of dietary fibers [31].

The substantial quantity of dietary fibers could be achieved by healthy populations through the consumption of natural plant foods and simultaneously lowering the intake of sugar and fats laden foods [31].

\section{Frequent intake of total plant foods}

There are arrays of available studies that validly support the beneficial roles of plant-based foods in the prevention of type 2 diabetes, thereby lowering the risks of related macrovascular and microvascular outcomes, as well as the management of type 2 diabetes patients, while through the combination of many processes, the intake of plant-based foods significantly helps in balancing blood glucose levels, in turn preventing the occurrence of type 2 diabetes [32].
The key plant-based foods components and regimens that offer these beneficial effects in diabetes prevention are vegetables, nuts, legumes, seeds, whole grains, and fruits, while simultaneously shunning and avoiding all food products from animal sources [32, 33].

In addition, the consumption of vegetarian foods, usually complete plant diets for example total grains, green vegetables, natural fruits, nuts, and legumes reduces the risk of diabetes, and helps in the management of the condition, while a positive risk relationship exists with consumption of meat and the risk of diabetes [34].

Recent findings from the appraisal of observational studies and randomized controlled trials studies on diet and prevention of type 2 diabetes suggested that substantial consumption of cereal fibers, reduced intake of saturated fats, higher intake of polyphenols (anthocyanins) and magnesium, and cessation or massive reduction in the consumption of sugar-laden foods and beverages offers significant benefits in the prevention of type 2 diabetes [35].

\section{Quitting and avoidance of smoking}

Active and passive smoking significantly increase the occurrence of diabetes and its complications [36].

There is minimal weight gain after smoking cessation at first and this increases the risk of diabetes, however, this risk is diminished after continuous cessation of smoking for longer years, while permanent smoking cessation is usually beneficial for the overall health [36].

The effects of smoking on diabetes patients and its etiological roles as a risk for complications are through different mechanisms [37]. To this end, early and higher mortality rates are recorded among diabetic smokers due to macrovascular complications compared to nondiabetic smokers because smoking increases microvascular complications and is also a catalyst for the advancement from normoglycaemia to impaired glucose tolerance status, thereby increasing the serum glucose levels which is the major risk factor for type 2 diabetes [37].

As observed by several researchers, there is a strong correlation between smoking and the incidence of diabetes and its complications, as well as cardiovascular problems among people with diabetes [38]. For example, in Japan, there is a $16 \%$ rise in the occurrence of type 2 diabetes for a daily smoking of 10 cigarettes, while $18.8 \%$ and $5.4 \%$ of type 2 diabetes diagnosed cases are caused by smoking in men and women respectively $[38,39]$.

\section{Maintaining a healthy weight}

Extra adiposity and concentrated serum glucose are significant risk factors for prediabetes because 
the progression and risk of type 2 diabetes is seven and twelve times higher for obese men and women respectively [40].

A substantial weight reduction via various lifestyle changes can diminish and prevent the occurrence of type 2 diabetes among pre-diabetics because every kilogram of shed body weight is commensurable with a $16 \%$ reduction in the future occurrence of diabetes, while voluntary and intended weight shedding correlates directly with $15 \%$ reduction in all-cause complications and deaths [40].

Furthermore, weight loss offers beneficial effects in preventing the advancement to type 2 diabetes in high-risk people, and also prevents cardiovascular risk outcomes due to balancing of the blood glucose levels which is sacrosanct to the emergence of the disease, while in type 2 diabetics, weight loss helps in balancing the blood glucose levels which correlates with reduction and lesser requirements for frequent intake of sugarreducing drugs [41].

In addition, without the combination and intake of drugs, weight loss of $\sim 10 \%$ alone helps in preventing the future incidence and progression of diabetes among high-risk people, and further beneficial effects will be achieved with a larger amount of weight loss due to the additional improvements on glucose equilibrium [41].

For weight loss to be effective in the prevention of diabetes, it must be consistent because the recurrence in the loss and gain of body weight (weight cycling) correlates significantly with a higher occurrence of diabetes [42].

Apart from lifestyle changes (dietary modifications and physical activity), the use of Livongo, a multifunctional mobile device that helps in tracking blood pressure, blood glucose levels, and weight information offers beneficial effects in improving blood glucose in type 2 diabetes patients, while an individualized lifestyle preventive coaching could be an additional beneficial intervention in controlling glucose levels and maintaining a healthy weight [43].

\section{Avoidance of alcohol consumption}

Excessive alcohol intake is a significant risk factor for the incidence of type 2 diabetes [44]. For instance, occasional drinkers have a significantly decreased risk of new-onset diabetes compared with none, regular, and heavy drinkers in Taiwan [44], while in China, higher consumption of alcohol was commeasurable with increased risk and complications of diabetes even among moderate men drinkers [45].

As is evident in many studies, low and moderate intakes of alcohol reduce the occurrence of diabetes, while heavy alcohol consumption correlates with increased incidence of diabetes in the future [46].
Likewise, alcohol intake exerts harmful health effects on the present and future wellbeing of young type 1 diabetes patients due to the major challenges in balancing the blood glucose levels [47].

\section{Avoidance of fats and sugars laden diets}

Extra adiposity through sugary and fatty foods strongly correlates with the incidence of non-insulindependent type 2 diabetes, while findings on lifestyle changes were impressive in reversing this occurrence [48].

The consumption of some specific fats offers beneficial effects in the prevention of type 2 diabetes, for instance, the intake of fish and marine $n-3$ fatty acids offered a protective role against the occurrence of type 2 diabetes in Asian people, while the intake of regular-fat dairy foods and trans-palmitoleic acid correlates with reduced incidence of type 2 diabetes among western populations [48].

Specifically, findings from an animal study on the effects of a moderate and high saturated fat diet on the incidence of diabetes-related pathologies revealed that high-caloric saturated fat consumption significantly induced diabetes in hamsters, possibly due to slowed secretion of insulin [49].

Furthermore, viable evidence affirms the protective role of polyunsaturated fatty acids in the prevention of type 2 diabetes, while the protective effects of monounsaturated and saturated fatty acids remain unclear [50].

As an alternative to fat diets, findings from a metaanalysis study recommend a likely protective effect of dairy food products, specifical yogurt in the prevention of type 2 diabetes [51].

\section{Avoiding drugs that induces diabetes}

Some specific medications that are used in the treatment of other health conditions for example beta-blockers, statins, corticosteroids, antipsychotics, and thiazide diuretics are associated with increased incidence of type 2 diabetes due to their role in increasing weight gain and blood glucose quantity. Although, these are usually reversed if such drugs are stopped and discontinued, while a smaller dosage prescription of these medications may also reduce the occurrence of concentrated blood glucose, diabetes, and weight gain [52].

In addition, some drugs are effective in the prevention of diabetes. For example, metformin offers a $29 \%$ preventive effect against the advancement of pre-diabetes to type 2 diabetes in high-risk populations [53].

Likewise, in combination with lifestyle modifications, and with caution for some specific individuals due to gastrointestinal complications, acarbose could 
offer beneficial effects in delaying the progression to type 2 diabetes in people with impaired glucose tolerance [54].

The ADA recommends the medical prescription of metformin for the prevention of diabetes in very highrisk populations [54].

\section{Controlling blood pressure and cardiovascular diseases}

Diabetes and cardiovascular disease have many similar risk factors which include adiposity and sedentary lifestyles, while cardiovascular disease is a significant cause of death in type 2 diabetes patients, and the rate of hypertension is substantially higher in type 2 diabetes patients compared to the general population $[55,56]$.

Likewise, hypertension exerts a greater risk for cardiovascular disease in diabetes patients, and blood pressure of $140 / 85 \mathrm{~mm} \mathrm{Hg}$ should be the minimal therapeutic goal for type 2 diabetes patients to reverse and reduce this effect [57].

Importantly, normal blood pressure could be achieved by the majority of people through a healthy diet, maintaining a healthy weight, and frequent physical activities, while some prescribed medications could offer beneficial effects in maintaining normal blood pressure, the option of medications should certainly be personalized with regards to the individual's age, gender, ethnicity and existing health conditions [56].

\section{Infection prevention}

Scientific information suggests the possible role of infection on insulin resistance through the proinflammatory cytokine response, the acute-phase response, and the alteration of the nutrient levels [58]. This suggests that any infection that causes or affects insulin resistance is a possible risk factor for type 2 diabetes [58].

Specifically, there is a significant association between $\mathrm{H}$. pylori infection and the incidence of type 2 diabetes via the effects on chronic inflammation, insulin resistance, and low insulin production due to the impairments of the pancreas $\beta$-cells, lipotoxicity, and glucotoxicity [59].

The aforementioned mechanism provides an understanding of the causative role of $\mathrm{H}$. pylori infection in the occurrence of type 2 diabetes, consequently the need for $H$. pylori and infections prevention, and eradication efforts to mitigate the occurrence of type 2 diabetes in high-risk populations $[59,60]$.

\section{Ensuring regular medical checkup and screening}

Type 2 diabetes, which causes morbidity, complications, and mortality in association with other health conditions is a major health problem confronting the primary health services universally [61].

The need for regular medical screening for type 2 diabetes is pertinent for early diagnosis because the majority of people are unaware, they have the disease globally [61]. The ADA recommends regular blood glucose screening for high-risk individuals, and these categories of people are 1) > 45 years of age, 2) been overweight and obese at any age, 3) prediabetes, 4) having inactive and sedentary lifestyles, and 5) having a family history of diabetes [62].

In addition, screening methods such as the oral glucose tolerance test, measuring fasting and 2-hours postprandial glucose values, the 1-hour glucose value, measuring the waist circumference, and EZSCAN ${ }^{\mathrm{TM}}$ technology based on reverse iontophoresis are some of the standardized and globally acceptable diabetes screening methods which have beneficial effects in preventing the progression of diabetes through the need for the adoption of lifestyle changes and modifications among high-risk people (prediabetes) [63].

\section{Prevention of depression and stress}

Depression significantly correlates with a $41 \%$ higher occurrence of diabetes, and 32\% higher odds for the occurrence of type 2 diabetes [64]. This supports the need for the inclusion of depressive manifestations as possible diabetes screening requirements which may help with the early detection of the condition $[64,65]$.

The types of depression that usually increase the risk of diabetes are non-severe, persistent and untreated depression [66]. Due to the deleterious health consequences that occur from the combination of diabetes and depression, all forms of depression must be prevented, and every issue of depression must the treated with antidepressants, although with adequate attention to age and other existing health conditions of the patients [66].

Stress is an established causative factor for the incidence of type 2 diabetes, and also plays a significant role in the progression of new-onset type 2 diabetes [67].

As explained elaborately by Sharma and colleague [68], the role of stress in the emergence and progression of type 2 diabetes is through multifaceted effects on the hypothalamic-pituitary-adrenal (HPA) axis (i.e., the stress response system) and the nervous system (sympathetic nervous system), while through a similar mechanism, stress is a significant factor in the origin and development of type 1 diabetes [69].

\section{Conclusions}

In respect to the yearly global surge in diabetes cases, and the predicted diabetes epidemic in the con- 
tinents of Africa and Asia in the future, and in addition to some COVID-19 mitigative measures which includes but are not limited to minimal and full lockdowns, specific restrictions, staying indoors, working from home and curfews which are likely to favor the practices of some diabetes risk factors such as sedentary lifestyle, inactivity, and unhealthy eating.

As a consequence, these leads to increases in diabetes cases during the pandemic periods. To this end, there is a prompt need for the provision and emphasis on efficient and viable diabetes prevention plan that is generally acceptable worldwide [70].

According to the current reviewed evidence, this author concludes that engaging in regular physical activity, adequate intake of dietary fiber, intake of total plant foods, quitting and avoiding smoking, maintaining a healthy weight, avoiding the intake of alcohol, avoiding fats and sugars laden diets, avoiding drugs that induce diabetes, controlling blood pressure and cardiovascular diseases, infection prevention, regular medical checkup and screening, and prevention of depression and stress will offer beneficial effects in the prevention of diabetes, preventing the progression of diabetes among the high-risk population, and the prevention of complications among diabetes patients during and after the pandemic.

Furthermore, during the pandemic period, it is essential for the general public and diabetes patients to adhere strictly to all the measures discussed in this article to enable the prevention of diabetes, which exerts severe outcomes with COVID-19, and improvement of the condition.

In addition, it is important for primary health care practitioners, various governmental health agencies and specific non-governmental organizations in all regions globally to intensify and sustain diabetes prevention efforts to prevent the projected future occurrences of the condition.

\section{Acknowledgements}

Great thanks and appreciations to my advisor Dr. R. Marks, Teachers College, Columbia University, New York, USA who helped to proofread the full manuscript for appropriateness and offered valuable suggestions. Also, I appreciate the authors of articles reviewed for impacting on my ideas in this write-up and anonymous reviewers of this great journal for their valuable comments that enriched the final copy published.

\section{Conflicts of interest}

None.

\section{REFERENCES}

1. World Health Organization. Diabetes. https://www.who.int/newsroom/fact-sheets/detail/diabetes (9.02.2020).

2. International Diabetes Federation. IDF DIABETES ATLAS Ninth Edition 2019. https://www.diabetesatlas.org/upload/resources/material/20200302_133351 IDFATLAS9e-final-web.pdf (8.02.2020).

3. John E. Diabetics worldwide 2019 and 2045. https://www. statista.com/statistics/271442/number-of-diabetics-worldwide/ (8.02.2019).

4. Shi $\mathrm{Y}, \mathrm{Hu}$ FB. The global implications of diabetes and cancer. Lancet. 2014; 383(9933): 1947-1948, doi: 10.1016/S01406736(14)60886-2, indexed in Pubmed: 24910221.

5. World Health Organization. Global Report on Diabetes. https:// apps.who.int/iris/bitstream/10665/204871/1/9789241565257_ eng.pdf\&sa $=$ U\&ved $=2$ ahUKEwjtqIW-pOjsAhUJ6aQKHZYh BqUQFjAAegQIBBAB\&usg = AOvVaw2dlq0L_Xe4wEd-yqDP011v (8.02.2020).

6. Melmed S, Larsen PR, Kronenberg H. Williams textbook of endocrinology (12th ed.). Elsevier/Saunders. 2011: 1371-1435.

7. Vos T, Flaxman AD, Naghavi $M$, et al. Years lived with disability (YLDs) for 1160 sequelae of 289 diseases and injuries 1990-2010: a systematic analysis for the Global Burden of Disease Study 2010. Lancet. 2012; 380(9859): 2163-2196, doi: 10.1016/S01406736(12)61729-2, indexed in Pubmed: 23245607.

8. Gale E, Gillespie KM. Diabetes and gender. Diabetologia. 2001; 44(1): 3-15, doi: 10.1007/s001250051573.

9. Meisinger C, Thorand B, Schneider A, et al. Sex differences in risk factors for incident type 2 diabetes mellitus: the MONICA Augsburg cohort study. Arch Intern Med. 2002; 162(1): 82-89, doi: 10.1001/archinte.162.1.82, indexed in Pubmed: 11784224.

10. Public Health Agency of Canada, Diabetes in Canada: Facts and figures from a public health perspective. Ottawa, 2011. https:// www.canada.ca/content/dam/phac-aspc/migration/phac-aspc/ cd-mc/publications/diabetes-diabete/facts-figures-faits-chiffres-2011/pdf/facts-figures-faits-chiffres-eng.pdf (8.02.2020).

11. Mathers CD, Loncar D. Projections of global mortality and burden of disease from 2002 to 2030. PLoS Med. 2006; 3(11): e442, doi: 10.1371/journal.pmed.0030442, indexed in Pubmed: 17132052.

12. Wild S, Roglic G, Green A, et al. Global prevalence of diabetes: estimates for the year 2000 and projections for 2030. Diabetes Care. 2004; 27(5): 1047-1053, doi: 10.2337/diacare.27.5.1047, indexed in Pubmed: 15111519.

13. American Diabetes Association. Economic Costs of Diabetes in the U.S. in 2017. Diabetes Care. 2018; 41(5): 917-928, doi: 10.2337/ dci18-0007, indexed in Pubmed: 29567642.

14. Center for Disease Control and Prevention. Deaths and Cost I Data \& Statistics | Diabetes . https://www.cdc.gov/nchs/nvss/ deaths.htm (8.02.2020).

15. Ayenigbara IO. COVID-19: An International Public Health Concern. Cent Asian J Glob Health. 2020; 9(1): e466, doi: 10.5195/ cajgh.2020.466, indexed in Pubmed: 33062403.

16. Center for Disease Control and Prevention. COVID-19. Clinical Care Guidance. [Internet]. 2020. https://www.cdc.gov/ coronavirus/2019-ncov/hcp/clinical-guidance-managementpatients.html (8.02.2020).

17. Miller LE, Bhattacharyya R, Miller AL. Diabetes mellitus increases the risk of hospital mortality in patients with Covid-19: Systematic review with meta-analysis. Medicine (Baltimore). 2020; 99(40): e22439, doi: 10.1097/MD.0000000000022439, indexed in Pubmed: 33019426.

18. Aggarwal G, Lippi G, Lavie CJ, et al. Diabetes mellitus association with coronavirus disease 2019 (COVID-19) severity and mortality: A pooled analysis. J Diabetes. 2020; 12(11): 851-855, doi: 10.1111/1753-0407.13091, indexed in Pubmed: 32677321. 
19. Ceriello A, Standl E, Catrinoiu D, et al. "Diabetes and Cardiovascular Disease (D\&CVD)" Study Group of the European Association for the Study of Diabetes (EASD). Issues for the management of people with diabetes and COVID-19 in ICU. Cardiovasc Diabetol. 2020; 19(1): 114, doi: 10.1186/s12933-020-01089-2, indexed in Pubmed: 32690029.

20. Nandy K, Salunke A, Pathak SK, et al. Coronavirus disease (COVID-19): A systematic review and meta-analysis to evaluate the impact of various comorbidities on serious events. Diabetes Metab Syndr. 2020; 14(5): 1017-1025, doi: 10.1016/j.dsx.2020.06.064, indexed in Pubmed: 32634716.

21. Bello-Chavolla OY, Bahena-López JP, Antonio-Villa NE, et al. Predicting Mortality Due to SARS-CoV-2: A Mechanistic Score Relating Obesity and Diabetes to COVID-19 Outcomes in Mexico. J Clin Endocrinol Metab. 2020; 105(8), doi: 10.1210/clinem/dgaa346, indexed in Pubmed: 32474598.

22. International Diabetes Federation. COVID-19 and Diabetes. [Internet]. 2021. https://www.idf.org/aboutdiabetes/whatis-diabetes/covid-19-and-diabetes/1-covid-19-and-diabetes. html (8.02.2020).

23. Aune $D$, Norat $T$, Leitzmann $M$, et al. Physical activity and the risk of type 2 diabetes: a systematic review and dose-response metaanalysis. Eur J Epidemiol. 2015; 30(7): 529-542, doi: 10.1007/ s10654-015-0056-z, indexed in Pubmed: 26092138.

24. Francesconi $C$, Niebauer J, Haber $P$, et al. [Physical activity and exercise training in the prevention and therapy of type 2 diabetes mellitus]. Wien Klin Wochenschr. 2016; 128 Suppl 2(Suppl 1): S141-S145, doi: 10.1007/s00508-015-0923-3, indexed in Pubmed: 27052239.

25. Lumb A. Diabetes and exercise. Clin Med (Lond). 2014; 14(6): 673-676, doi: 10.7861/clinmedicine.14-6-673, indexed in Pubmed: 25468857.

26. Aune D, Sen A, Henriksen T, et al. Physical activity and the risk of gestational diabetes mellitus: a systematic review and doseresponse meta-analysis of epidemiological studies. Eur J Epidemiol. 2016; 31(10): 967-997, doi: 10.1007/s10654-016-0176-0, indexed in Pubmed: 27485519.

27. Di Biase N, Balducci S, Lencioni C, et al. Review of general suggestions on physical activity to prevent and treat gestational and pre-existing diabetes during pregnancy and in postpartum. Nutr Metab Cardiovasc Dis. 2019; 29(2): 115-126, doi: 10.1016/j. numecd.2018.10.013, indexed in Pubmed: 30642790.

28. Weickert MO, Pfeiffer AFH. Impact of Dietary Fiber Consumption on Insulin Resistance and the Prevention of Type 2 Diabetes. J Nutr. 2018; 148(1): 7-12, doi: 10.1093/jn/nxx008, indexed in Pubmed: 29378044

29. Sylvetsky AC, Edelstein SL, Walford G, et al. Diabetes Prevention Program Research Group. A High-Carbohydrate, High-Fiber, Low-Fat Diet Results in Weight Loss among Adults at High Risk of Type 2 Diabetes. J Nutr. 2017; 147(11): 2060-2066, doi: 10.3945/ jn.117.252395, indexed in Pubmed: 28954840.

30. Weickert MO. High fiber intake, dietary protein, and prevention of type 2 diabetes. Expert Rev Endocrinol Metab. 2018; 13: 223-224.

31. DahI WJ, Stewart ML. Position of the Academy of Nutrition and Dietetics: Health Implications of Dietary Fiber. J Acad Nutr Diet. 2015; 115(11): 1861-1870, doi: 10.1016/j.jand.2015.09.003, indexed in Pubmed: 26514720.

32. Joshi S, McMacken M, Kalantar-Zadeh K, et al. A plant-based diet for the prevention and treatment of type 2 diabetes. J Geriatr Cardiol. 2017; 14(5): 342-354, doi: 10.11909/j.issn.16715411.2017.05.009, indexed in Pubmed: 28630614.

33. Schwingshackl L, Hoffmann G, Lampousi AM, et al. Food groups and risk of type 2 diabetes mellitus: a systematic review and meta-analysis of prospective studies. Eur J Epidemiol. 2017; 32(5): 363-375, doi: 10.1007/s10654-017-0246-y, indexed in Pubmed: 28397016.

34. Olfert MD, Wattick RA. Vegetarian Diets and the Risk of Diabetes. Curr Diab Rep. 2018; 18(11): 101, doi: 10.1007/s11892-018-10709, indexed in Pubmed: 30229314.
35. Palacios OM, Kramer M, Maki KC. Diet and prevention of type 2 diabetes mellitus: beyond weight loss and exercise. Expert Rev Endocrinol Metab. 2019; 14(1): 1-12, doi: 10.1080/174466 51.2019.1554430, indexed in Pubmed: 30521416.

36. Brath $\mathrm{H}$, Kaser $\mathrm{S}$, Tatschl C, et al. [Smoking, alcohol and diabetes (Update 2019)]. Wien Klin Wochenschr. 2019; 131(Suppl 1): 67-70, doi: 10.1007/s00508-019-1455-z, indexed in Pubmed: 30980165.

37. Śliwińska-Mossoń M, Milnerowicz H. The impact of smoking on the development of diabetes and its complications. Diab Vasc Dis Res. 2017; 14(4): 265-276, doi: 10.1177/1479164117701876, indexed in Pubmed: 28393534.

38. Zhu P, Pan XF, Sheng L, et al. Cigarette Smoking, Diabetes, and Diabetes Complications: Call for Urgent Action. Curr Diab Rep. 2017; 17(9): 78, doi: 10.1007/s11892-017-0903-2, indexed in Pubmed: 28766247.

39. Akter S, Goto A, Mizoue T. Smoking and the risk of type 2 diabetes in Japan: A systematic review and meta-analysis. J Epidemiol. 2017; 27(12): 553-561, doi: 10.1016/j.je.2016.12.017, indexed in Pubmed: 28716381.

40. Lau DCW, Teoh H. Current and Emerging Pharmacotherapies for Weight Management in Prediabetes and Diabetes. Can J Diabetes. 2015; 39 Suppl 5: S134-S141, doi: 10.1016/j. jcjd.2015.10.001, indexed in Pubmed: 26654857.

41. Grams J, Garvey WT. Weight Loss and the Prevention and Treatment of Type 2 Diabetes Using Lifestyle Therapy, Pharmacotherapy, and Bariatric Surgery: Mechanisms of Action. Curr Obes Rep. 2015; 4(2): 287-302, doi: 10.1007/s13679-015-0155-x, indexed in Pubmed: 26627223.

42. Rhee EJ, Cho JH, Kwon H, et al. Increased risk of diabetes development in individuals with weight cycling over 4 years: The Kangbuk Samsung Health study. Diabetes Res Clin Pract. 2018; 139: 230-238, doi: 10.1016/j.diabres.2018.03.018, indexed in Pubmed: 29574105.

43. Bollyky JB, Bravata D, Yang J, et al. Remote Lifestyle Coaching Plus a Connected Glucose Meter with Certified Diabetes Educator Support Improves Glucose and Weight Loss for People with Type 2 Diabetes. J Diabetes Res. 2018; 2018: 3961730, doi: 10.1155/2018/3961730, indexed in Pubmed: 29888288.

44. Lai YJ, Hu HY, Lee $Y L$, et al. Frequency of alcohol consumption and risk of type 2 diabetes mellitus: A nationwide cohort study. Clin Nutr. 2019; 38(3): 1368-1372, doi: 10.1016/j. clnu.2018.06.930, indexed in Pubmed: 30448092.

45. Peng $\mathrm{M}$, Zhang J, Zeng $\mathrm{T}$, et al. Alcohol consumption and diabetes risk in a Chinese population: a Mendelian randomization analysis. Addiction. 2019; 114(3): 436-449, doi: 10.1111/ add.14475, indexed in Pubmed: 30326548.

46. Polsky S, Akturk HK. Alcohol Consumption, Diabetes Risk, and Cardiovascular Disease Within Diabetes. Curr Diab Rep. 2017; 17(12): 136, doi: 10.1007/s11892-017-0950-8, indexed in Pubmed: 29103170.

47. MacNaught $N$, Holt P. Type 1 diabetes and alcohol consumption. Nurs Stand. 2015; 29(50): 41-47, doi: 10.7748/ns.29.50.41. e9812, indexed in Pubmed: 26264336.

48. Rice Bradley BH. Dietary Fat and Risk for Type 2 Diabetes: a Review of Recent Research. Curr Nutr Rep. 2018; 7(4): 214-226, doi: 10.1007/s13668-018-0244-z, indexed in Pubmed: 30242725.

49. Popov D, Simionescu M, Shepherd PR. Saturated-fat diet induces moderate diabetes and severe glomerulosclerosis in hamsters. Diabetologia. 2003; 46(10): 1408-1418, doi: 10.1007/ s00125-003-1185-6, indexed in Pubmed: 12898005.

50. Carrasquilla GD, Jakupović $H$, Kilpeläinen TO. Dietary Fat and the Genetic Risk of Type 2 Diabetes. Curr Diab Rep. 2019; 19: 109.

51. Gijsbers L, Ding EL, Malik VS, et al. Consumption of dairy foods and diabetes incidence: a dose-response meta-analysis of observational studies. Am J Clin Nutr. 2016; 103(4): 1111-1124, doi: 10.3945/ajcn.115.123216, indexed in Pubmed: 26912494. 
52. Diabetes.co.uk. Drug Induced Diabetes. [Internet]. 2020. https:// www.diabetes.co.uk/drug-induced-diabetes.html (8.02.2020).

53. Aroda VR, Knowler WC, Crandall JP, et al. Diabetes Prevention Program Research Group. Metformin for diabetes prevention: insights gained from the Diabetes Prevention Program/Diabetes Prevention Program Outcomes Study. Diabetologia. 2017; 60(9): 1601-1611, doi: 10.1007/s00125-017-4361-9, indexed in Pubmed: 28770322.

54. Chiasson JL, Josse RG, Gomis R, et al. STOP-NIDDM Trail Research Group. Acarbose for prevention of type 2 diabetes mellitus: the STOP-NIDDM randomised trial. Lancet. 2002; 359(9323): 2072-2077, doi: 10.1016/S0140-6736(02)08905-5, indexed in Pubmed: 12086760 .

55. Strain WD, Paldánius PM. Diabetes, cardiovascular disease and the microcirculation. Cardiovasc Diabetol. 2018; 17(1): 57, doi: 10.1186/s12933-018-0703-2, indexed in Pubmed: 29669543.

56. Vargas-Uricoechea H, Cáceres-Acosta MF. Blood pressure control and impact on cardiovascular events in patients with type 2 diabetes mellitus: A critical analysis of the literature. Clin Investig Arterioscler. 2019; 31(1): 31-47, doi: 10.1016/j.arteri.2018.07.001, indexed in Pubmed: 30274771.

57. Ferrannini E, Cushman WC. Diabetes and hypertension: the bad companions. Lancet. 2012; 380(9841): 601-610, doi: 10.1016/ S0140-6736(12)60987-8, indexed in Pubmed: 22883509.

58. Chakraborty S, Bhattacharyya R, Banerjee D. Infections: A Possible Risk Factor for Type 2 Diabetes. Adv Clin Chem. 2017; 80: 227-251, doi: 10.1016/bs.acc.2016.11.004, indexed in Pubmed: 28431641.

59. He C, Yang Z, Lu NH. Helicobacter pylori infection and diabetes: is it a myth or fact? World J Gastroenterol. 2014; 20(16): 4607-4617, doi: 10.3748/wjg.v20.i16.4607, indexed in Pubmed: 24782613

60. Kato M, Toda A, Yamamoto-Honda R, et al. Association between Helicobacter pylori infection, eradication and diabetes mellitus. J Diabetes Investig. 2019; 10(5): 1341-1346, doi: 10.1111/ jdi.13011, indexed in Pubmed: 30663265.
61. Martinez LC, Sherling D, Holley A. The Screening and Prevention of Diabetes Mellitus. Prim Care. 2019; 46(1): 41-52, doi: 10.1016/j. pop.2018.10.006, indexed in Pubmed: 30704659.

62. Shealy KM, Wu J, Waites J, et al. Patterns of Diabetes Screening and Prediabetes Treatment during Office Visits in the US. J Am Board Fam Med. 2019; 32(2): 209-217, doi: 10.3122/ jabfm.2019.02.180259, indexed in Pubmed: 30850457.

63. Schwarz PEH. [Screening and prevention of diabetes]. Internist (Berl). 2015; 56: 1124-33.

64. Yu M, Zhang X, Lu F, et al. Depression and Risk for Diabetes: A Meta-Analysis. Can J Diabetes. 2015; 39(4): 266-272, doi: 10.1016/j. jcjd.2014.11.006, indexed in Pubmed: 25773933.

65. Rotella F, Mannucci E, Rotella F, et al. Diabetes mellitus as a risk factor for depression. A meta-analysis of longitudinal studies. Diabetes Res Clin Pract. 2013; 99(2): 98-104, doi: 10.1016/j. diabres.2012.11.022, indexed in Pubmed: 23265924.

66. Campayo A, Gómez-Biel CH, Lobo A. Diabetes and depression. Curr Psychiatry Rep. 2011; 13(1): 26-30, doi: 10.1007/s11920010-0165-z, indexed in Pubmed: 21052874.

67. Hackett RA, Steptoe A. Type 2 diabetes mellitus and psychological stress - a modifiable risk factor. Nat Rev Endocrinol. 2017; 13(9): 547-560, doi: 10.1038/nrendo.2017.64, indexed in Pubmed: 28664919.

68. Sharma VK, Singh TG. Chronic Stress and Diabetes Mellitus: Interwoven Pathologies. Curr Diabetes Rev. 2020; 16(6): 546-556, doi: 10.2174/1573399815666191111152248, indexed in Pubmed: 31713487.

69. Sharif K, Watad A, Coplan L, et al. Psychological stress and type 1 diabetes mellitus: what is the link? Expert Rev Clin Immunol. 2018; 14(12): 1081-1088, doi: 10.1080/1744666X.2018.15387 87, indexed in Pubmed: 30336709.

70. Thomas T, Pfeiffer AFH. Foods for the prevention of diabetes: how do they work? Diabetes Metab Res Rev. 2012; 28(1): 25-49, doi: 10.1002/dmrr.1229, indexed in Pubmed: 21695766. 